\title{
Epigénesis: secuelas de una infancia adversa
}

\author{
Sofia Gonzalez Salinas, Sergio Manuel Sánchez Moguel y \\ Nelida Ramírez Jiménez
}

\begin{abstract}
Resumen
El maltrato infantil es un problema de salud pública a nivel mundial cuyas secuelas a largo plazo han sido poco descritas. Diversos estudios demuestran que una infancia adversa predispone al desarrollo de enfermedades cardiovasculares, metabólicas, inmunológicas y psiquiátricas en la edad adulta. Pero ¿cuál es el mecanismo que permite observar estos cambios patológicos varias décadas después de que ocurrieron los eventos adversos? Una posible respuesta reside en las modificaciones epigenéticas del genoma de las células, las cuales influyen en la expresión de la información hereditaria sin alterar la secuencia de nucleótidos del genoma. Los estudios más recientes sugieren que los cambios en la metilación del material genético son uno de los mecanismos epigenéticos a través del cual las historias de maltrato dejan huellas en la salud física y emocional del adulto.
\end{abstract}

Palabras clave: infancia, maltrato, epigénesis, ADN, metilación.

\section{EPIGENESIS: CONSEQUENCES OF AN ADVERSE CHILDHOOD}

\begin{abstract}
Maltreatment during early-life is a world health problem whose long-term consequences have been poorly described. Multiple studies show that an adverse childhood increases the risk of developing cardiovascular, metabolic, inmunologic and psiquiatric disorders in adulthood. However, how is it that several years after the adverse event physiological and behavioral alterations can still be observed? A possible explanation relies on epigenetic alterations in the cells' genome, which affects the expression of hereditary information without changing its nucleotide sequence. Recent studies suggest that changes in methylation patterns of genetic material are one of the epigenetic mechanisms through which an adverse infancy leaves a footprint in an adult's physical and emotional health.
\end{abstract}

Keywords: childhood, child-abuse, epigenesis, DNA, methylation.

DOI: http://doi.org/10.22201/codeic.16076079e.2019.v20n2.a1 


\section{Sofia Gonzalez Salinas}

Investigadora Titular A en el área de Medicina y Coordinadora de la Licenciatura en Médico Cirujano de la Escuela Superior Tepeji del Río, Universidad Autónoma del Estado de Hidalgo. Candidata a Investigador Nacional. Miembro de la Society for Neuroscience y de la Sociedad Mexicana de Ciencias Fisiológicas.

\section{Sergio Manuel Sánchez Moguel}

smanuel sanchez@uaeh.edu.mx

Investigador Titular de Tiempo Completo en el área de Psicología de la Escuela Superior de Atotonilco de Tula, Universidad Autónoma del Estado de Hidalgo. Miembro de la Society for Neuroscience. Candidato a doctor en Psicología (Neurociencias de la conducta). Obtuvo la maestría en Ciencias (Neurobiología), ambos por la Universidad Nacional Autónoma de México (UNAM), Campus Juriquilla, en el Instituto de Neurobiología. Realizó la licenciatura en Psicología en la Universidad Autónoma de Campeche y la Licenciatura en Arquitectura en el Instituto Tecnológico de Campeche.

\section{Nelida Ramírez Jiménez}

754nelida8@gmail.com

Estudiante de cuarto semestre de la licenciatura en Tecnología del Centro de Física Aplicada y Tecnología Avanzada de la UNAM, Campus Juriquilla. 


\section{Introducción}

La Organización Mundial de la Salud reconoce que el maltrato infantil es un problema de salud pública a nivel mundial (Butchart, Harvey, Mian, y Fürniss, 2009). En México, alrededor de 13\% de los niños entre 6 y 9 años indican que son maltratados en su hogar (Hernández Dávila, 2013). Por otra parte, se ha reportado que $42.2 \%$ de mujeres jóvenes sufrieron violencia física durante la niñez, e incluso cuando estaban embarazadas (Cuevas, Blanco, Juárez, Palma, y Valdez-Santiago, 2006; Olaiz, Rojas, Valdez, Franco, y Palma, 2006). El maltrato a las mujeres embarazadas provoca daños también en el feto, ya que estos bebés presentan mayores complicaciones durante y después del parto y tienen un bajo peso al nacer (Valdez-Santiago y Sanín-Aguirre, 1996); como veremos más adelante, el maltrato infantil podría incluso iniciar desde el útero de la madre.

¿Cómo se establece este vínculo entre lo que ocurrió en los primeros años de vida con afecciones en la edad adulta? La hipótesis de Barker propone que las

Trabajos recientes muestran que una pobre calidad de vida durante la infancia se vincula con cambios epigenéticos en el adolescente y adulto. enfermedades del adulto tienen un origen fetal. Esta hipótesis se fundamentó en la observación de que un bajo peso al nacer se relacionaba con problemas de salud en el adulto (Calkins y Devaskar, 2011). La hipótesis del origen fetal de las enfermedades establece que las afecciones en el adulto son probablemente debidas a etapas "plásticas" durante el desarrollo, en las cuales el feto puede tener cambios en respuesta a su entorno (Calkins y Devaskar, 2011). La hipótesis de Barker ha ayudado a entender por qué el organismo responde de manera anómala cuando hubo antecedentes de maltrato infantil. Un elemento importante para explicar los cambios a largo plazo de una infancia adversa son las modificaciones epigenéticas. Este tema es poco conocido no sólo en la población en general sino también en las ciencias biológicas y de la salud; sin embargo, su entendimiento provee la posibilidad de desarrollar nuevos tratamientos para evitar que una infancia adversa deje huellas en la edad adulta.

\section{Alteraciones fisiológicas y psicológicas provocadas por una pobre calidad de vida durante la infancia}

Primero, es importante concebir el impacto que tiene el maltrato infantil en la salud física y mental del adulto para después entender procesos que ocurren a nivel celular, tales como la epigénesis, que podrían ser los responsables de mantener a largo plazo las alteraciones en la salud. 
"Epigénesis: secuelas de una infancia adversa" Sofia Gonzalez Salinas, Sergio Manuel Sánchez Moguel y Nelida Ramírez Jiménez

Las alteraciones fisiológicas (cambios en el funcionamiento de órganos y sistemas) relacionadas con una pobre calidad de vida durante el período de gestación o infancia se pueden clasificar en: 1) cardiometabólicas, 2) inmunitarias, 3) neuroanatómicas y 4) hormonales. Dentro de las alteraciones cardiometabólicas se ha encontrado que los adultos que vivieron situaciones estresantes durante el período de gestación o infancia — tales como vivir en un entorno de bajo nivel socioeconómico o cuyas madres sufrieron un divorcio durante el embarazo, tuvieron problemas de pareja, o presenciaron alguna catástrofe natural o social- presentan con mayor frecuencia diagnósticos de diabetes, obesidad, altos niveles de colesterol, niveles elevados de glucosa, alteraciones en la coagulación sanguínea, enfermedades coronarias, problemas renales, circulatorios y respiratorios (Entringer, Wüst et al., 2008; Lin y Liu, 2014; Roseboom, Painter, van Abeelen, Veenendaal y de Rooij, 2011; Sotomayor, 2013; Tamayo, Christian y Rathmann, 2010).

Con respecto al funcionamiento del sistema inmune, un estudio encontró que éste responde de manera incrementada en mujeres adultas cuyas madres sufrieron eventos negativos durante el embarazo - por ejemplo, conflictos de pareja, enfermedad grave o muerte de alguien cercano, problemas financieros, desaprobación familiar, etcétera-(Entringer, Kumsta et al., 2008). Una respuesta aumentada del sistema inmune también se ha observado en niños con estatus socioeconómico bajo (Azad et al., 2012).

Cambios en la composición y tamaño del cerebro también se relacionan con una infancia adversa. Un estudio que incluyó a adolescentes que vivían con familias adoptivas, pero que durante su infancia vivieron en orfanatorios rumanos, donde la calidad de atención era muy baja, mostró un menor porcentaje tanto de sustancia blanca (axones neuronales) como de sustancia gris cerebral (somas neuronales) con respecto a adolescentes que siempre vivieron con su familia biológica (Mehta et al., 2009). La amígdala es una región del cerebro localizada en el lóbulo temporal, ampliamente vinculada con la regulación de emociones de tipo negativo. Diversos estudios muestran que los niños que vivieron en orfanatos presentan un mayor tamaño de amígdala (Mehta et al., 2009; Tottenham et al., 2010). Incluso se ha encontrado una correlación directa entre el tamaño de amígdala y el tiempo que vivieron en los orfanatos antes de ser adoptados (Tottenham et al., 2010); un mayor tamaño se correlaciona a su vez con menor capacidad de regular las emociones, mayores niveles de ansiedad y mayor incidencia de desórdenes psiquiátricos (Tottenham et al., 2010). Además de la amígdala, otras regiones cerebrales que presentan un tamaño reducido, relacionado con una baja calidad de vida durante la infancia, son la corteza anterior cingulada, la corteza prefrontal y el núcleo caudado (Cohen et al., 2006; Tomoda et al., 2009), las cuales son importantes en procesos como el control inhibitorio, la atención y la toma de decisiones.

La respuesta fisiológica ante una situación estresante o de peligro está guiada por el eje hipotálamo-pituitaria-adrenal (eje HPA), el cual regula la 


\begin{abstract}
producción y liberación de hormonas del estrés tales como la adrenocorticotropa, la hormona liberadora de corticotropina, cortisol, corticosterona, adrenalina y noradrenalina. Las niñas y adolescentes que sufrieron abuso sexual muestran alteraciones basales en el eje HPA, pero su sistema hormonal responde de manera adecuada cuando es estimulado (De Bellis et al., 1994). Sin embargo, mujeres adultas con antecedentes de maltrato infantil muestran una respuesta incrementada del eje HPA ante una situación de estrés, la respuesta fue aún mayor en aquellas con síntomas de depresión o ansiedad (Heim et al., 2000). En la figura 1 se resumen las alteraciones fisiológicas observadas en la adultez como consecuencia de experiencias adversas en la infancia.
\end{abstract}

\begin{tabular}{|c|c|}
\hline $\begin{array}{l}\text { Alteraciones fisiológicas } \\
\text { - Enfermedades metabólicas } \\
\text { Entringer, Wüst et al., 2008; } \\
\text { Roseboom et al., 2011; Sotomayor, } \\
\text { 2013; Tamayo et al., } 2010 . \\
\text { - Problemas circulatorios } \\
\text { Lin y Liu, 2014; Roseboom et al., } \\
\text { 2011; Sotomayor, } 2013 . \\
\text { - Afecciones respiratorias y renales } \\
\text { Lin y Liu, } 2014 . \\
\text { - Mayor respuesta inmune } \\
\text { Azad et al., 2012; Entringer et al., } \\
\text { 2008. } \\
\text { Cambios en la estructura cerebral } \\
\text { Cohen et al., 2006; Mehta et al., } \\
\text { 2009; Tomoda et al., 2009; } \\
\text { Tottenham et al., } 2010 . \\
\text { Alteraciones en la respuesta } \\
\text { hormonal de estrés } \\
\text { De Bellis et al., 1994. }\end{array}$ & $\begin{array}{l}\text { Alteraciones psicológicas } \\
\text { - Conducta suicida } \\
\text { De Bellis et al., 1994; Iram Rizvi y } \\
\text { Najan et al., 2014. } \\
\text { - Altos niveles de ansiedad } \\
\text { Downs y Rindels, 2004; Iram Rizvi } \\
\text { y Najan et al., 2014; Springer et al., } \\
2007 . \\
\text { - Depresión } \\
\text { Downs y Rindels, 2004; Negele et } \\
\text { al., 2015; Springer et al., } 2007 . \\
\text { - Problemas de conducta } \\
\text { Iram Rizvi y Najan et al., } 2014 . \\
\text { - Alteraciones de sueño } \\
\text { Downs y Rindels, 2004. } \\
\text { - Habilidades cognoscitivas } \\
\text { pobres } \\
\text { Beckett et al., } 2006 .\end{array}$ \\
\hline
\end{tabular}

Figura 1. Alteraciones en el adulto relacionadas con experiencias adversas en la infancia.
En el aspecto psicológico se ha encontrado que los niños y adolescentes que sufren abuso sexual o de tipo psicológico tienen con frecuencia ideas suicidas, mayores intentos de suicidio, presentan distimia —un tipo de depresión crónica-, tienen problemas afectivos, de ansiedad, somáticos, de atención e hiperactividad, y de conducta; incluso, los adolescentes que percibieron a sus padres como más abusivos mostraron mayores problemas psicológicos (De Bellis et al., 1994; Espinoza-Gómez et al., 2010; Iram Rizvi y Najam, 2014; Páramo Castillo y Chávez Hernández, 2007; Pérez-Amezcua et al., 2010). Sin embargo, estos problemas de la infancia no se quedan en esa etapa; adultos con antecedentes de infancia adversa también presentan problemas de ansiedad, depresión, disociación y alteraciones del sueño (Downs y Rindels, 2004; Negele, Kaufhold, Kallenbach y Leuzinger-Bohleber, 2015; Springer, Sheridan, Kuo, y Carnes, 2007). Adicionalmente, algunos trabajos muestran que mayores afecciones en 
la salud mental del adulto se relacionan con eventos adversos en la infancia en comparación de la ocurrencia de éstos en la edad adulta (McCauley et al., 1997; Shrira, Shmotkin, y Litwin, 2012; Zlotnick, Warshaw, Shea, y Keller, 1997).

Apesar de las experiencias adversas, Iosniños pueden recuperar habilidades mentales dependiendo de la edad en la que se realice la intervención. En el trabajo de Beckett et al. (2006) se estudiaron niños que vivieron en orfanatos de Rumania y que posteriormente fueron adoptados en Reino Unido. Los niños que vivieron en orfanatos rumanos sólo 6 meses antes de ser adoptados mostraron un desarrollo intelectual adecuado para su edad de estudio (11 años), mientras que los niños que permanecieron más de 24 meses en instituciones de Rumania antes de ser adoptados en Reino Unido mostraron una menor capacidad intelectual. Estos resultados son altamente promisorios, ya que indican que la huella de maltrato es reversible si se interviene en el momento adecuado. Sin embargo, aún no se conocen los detalles de las terapias más efectivasy del alcance de las mejorías con respecto a la predisposición al desarrollo de enfermedades cardiovasculares, funcionamiento del sistema inmune, funcionamiento del eje HPA y de la composición cerebral.

\section{Epigénesis: hacia el ADN y más allá}

Ahora que hemos explorado el impacto que tiene una infancia adversa en la salud del niño y del adulto, tratemos de entender las bases celulares que subyacen a esta huella de maltrato. Si colocamos la palabra "epigénesis" en cualquier buscador en internet encontraremos definiciones relacionadas con el desarrollo de órganos. La epigénesis "molecular" a la que nos queremos referir en este artículo dista mucho de estas definiciones, vamos a ver que prácticamente es la responsable de tener varios tipos de célula en nuestro cuerpo y finalmente distintos órganos y sistemas.

Podemos definir la epigénesis como las marcas químicas reversibles que se añaden al material genético y que no alteran su composición nucleotídica, pero que sí influyen en su expresión. Vayamos ahora explicando cada parte de esta definición. El material genético se refiere a todo el ácido desoxirribonucleico (ADN) localizado en una célula, principalmente en el núcleo celular — no hay que pasar por alto que en la mitocondria también hay ADN y que también tiene marcas epigenéticas (Ghosh, Singh, Sengupta y Scaria, 2015)_. El ADN se conforma por la unión de los nuclétidos adenina, timina, citosina y guanina, por fosfatos y por un azúcar llamada desoxirribosa; estos elementos forman dos cadenas que corren en sentido opuesto (para conocer más sobre la estructura e historia del ADN revisar Gil Trejo, Laureano Viveros, y González-Salinas, 2018; GonzálezSalinas, 2018). A su vez, el material genético se compone por regiones codificantes o genes y regiones no codificantes que incluyen zonas regulatorias. Para su correcto empaquetamiento dentro de la célula, el ADN se enrolla en un conjunto de proteínas denominadas histonas (jsi lo extendiéramos el ADN mediría 2 metros 
Figura 2. Cambios epigenéticos producidos por experiencias adversas.

En las células del organismo la información genética está contenida en los cromosomas. Esta información puede sufrir cambios epigenéticos como la metilación de ADN y acetilación de histonas.

Trabajos recientes muestran que una pobre calidad de vida durante la infancia se vincula con cambios epigenéticos en el adolescente y adulto (Borghol et al., 2012; Essex et al., 2013; Labonté et al., 2012; Provençal et al., 2014; Tobi et al., 2009). y una célula mide 100,000 veces menos!). A esta unión de ADN e histonas se le conoce como cromatina (ver figura 2). A los genes y a las histonas se les pueden añadir modificaciones epigenéticas que, si bien no alteran la secuencia del ADN 0 de las histonas, sí pueden alterar cómo se expresa determinado gen. Con expresión del gen nos referimos al producto que se genera a partir de él, que corresponde primero a un ácido ribonucleico (ARN), de tipo mensajero, que "transmite" la información que posteriormente se traduce en una proteína. Los ARN mensajeros (ARNm) son una copia de una sola cadena del ADN y están compuestos por las bases nitrogenadas adenina, uracilo, citosina y guanina, por fosfatos y el azúcar llamada ribosa y en conjunto forman un cadena sencilla. Por otra parte, las proteínas son los caballitos de batalla de la célula ya que participan en muchos procesos como producción de nuevas proteínas, crecimiento celular, división celular, replicación del material genético, actividad metabólica, entre otras.

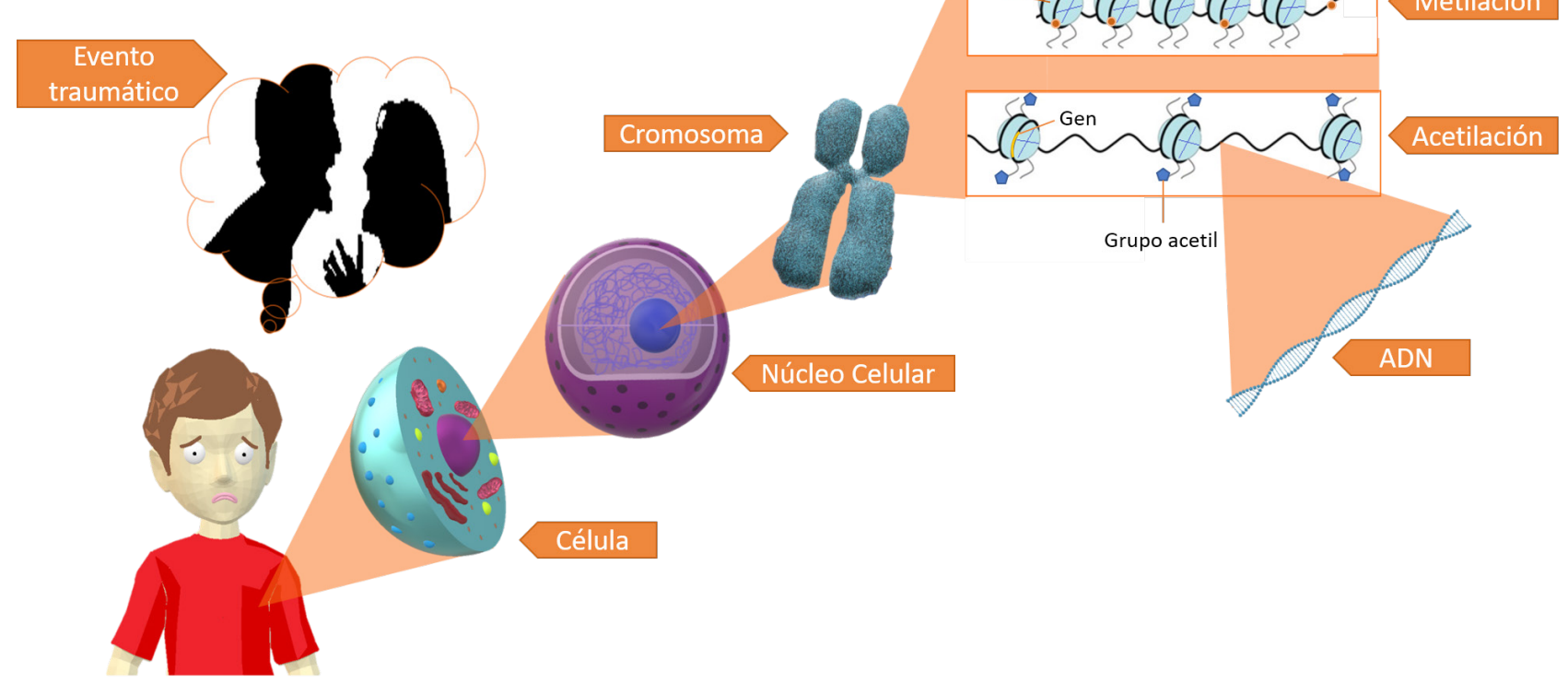

El principal cambio epigenético que sufre el ADN es la metilación que consiste en la adición de grupos metilo $\left(\mathrm{CH}_{3}\right)$ en regiones ricas en citosinas y guaninas conocidas como islas cpg. Comúnmente la metilación del ADN se asocia con la represión de la expresión génica. Dentro de las modificaciones que sufren las histonas tenemos la acetilación, fosforilación, metilación, ubiquitinación, y sumoilación. A pesar de que aún no se encuentra un código de combinaciones en las histonas que nos indique con claridad el efecto de dichas modificaciones sobre la expresión génica, se sabe bien que la acetilación se relaciona con mayor expresión. Los cambios epigenéticos son reversibles y se valen de diversas enzimas que agregan o quitan las respectivas modificaciones químicas (Delgado-Coello, 2011). Entonces, la expresión de los genes en una 
"Epigénesis: secuelas de una infancia adversa"

determinada región dependerá de las marcas epigenéticas localizadas en ella y, subsecuentemente, la cantidad de ARnm disponible y de proteína. El conjunto de genes expresados en determinadas células va a permitir que ciertas proteínas estén disponibles para su funcionamiento; es este particular grupo de proteínas lo que confiere la identidad y función a los tipos celulares y tejidos. Veamos un ejemplo, la rodopsina es una proteína encargada de captar la luz del ambiente y así transmitir a nuestro cerebro información de lo que observamos. Esta proteína se encuentra en altas concentraciones en las células de la retina y se corresponde con un bajo porcentaje de metilación de su gen mientras que el porcentaje de metilación de este mismo gen es muy alto en células del riñón o del cerebro y la cantidad de rodopsina en esos tejidos es prácticamente nula (Merbs et al., 2012).

En los últimos años, múltiples grupos de investigación han inspirado su trabajo en el estudio de los cambios epigenéticos que ocurren en el cerebro y su relación con el estrés, el envejecimiento y enfermedades mentales (Murgatroyd y Spengler, 2011). Durante períodos críticos como la infancia, procesos como el aprendizaje, almacenamiento y formación de la memoria, lenguaje y habilidades motrices tienen un crecimiento exponencial (Taliaz, 2013). A estas habilidades les subyacen etapas del desarrollo cerebral que involucran la generación de nuevas neuronas, migración de neuronas a distintas partes del cerebro, crecimiento de axones y dendritas (prolongaciones de las neuronas), formación de nuevas conexiones entre neuronas (sinápsis), muerte celular, eliminación de algunas conexiones sinápticas y formación de mielina en los axones neuronales importante para la propagación del impulso eléctrico a lo largo del axón de la neurona- (Kolb, Mychasiuk, Muhammad y Gibb, 2013). Estos cambios deben ocurrir en momentos, lugares y en cantidades precisas, ya que alteraciones en estas etapas se han vinculado con el desarrollo de desórdenes como el autismo, epilepsia o esquizofrenia (Mochida y Walsh, 2004).

Justo después de la fertilización del óvulo, se establecen marcas epigenéticas en algunos de los genes del nuevo ser siguiendo el patrón de marcas encontradas en los padres, a estas primeras marcas se les conoce como impronta genómica. El cerebro expresa particularmete un alto número de genes que sufren impronta genómica y se ha vinculado a este conjunto de genes con el desarrollo del cerebro, la conducta reproductiva, las emociones y la cognición (Ho-Shing y Dulac, 2019). Adicionalmente, se ha encontrado que el establecimiento de las marcas de metilación del ADN en una región del cerebro llamada corteza frontal ocurre durante los primeros años de vida y hasta los primeros años de la edad adulta coincidiendo con la formación de conexiones neuronales (Lister et al., 2013). Además, se ha reportado que las neuronas tienen una alta proporción de metilación no canónica en donde se agregan grupos metilo a los pares de nucleótidos CA, CC O ct en lugar de los nucleótidos CG (Lister et al., 2013); la relevancia de este patrón inusual de metilación aún está siendo estudiado. 
"Epigénesis: secuelas de una infancia adversa"

\section{Papel de la epigénesis en las alteraciones causadas por experiencias tempranas adversas}

Los primeros estudios que vincularon las experiencias adversas tempranas con cambios epigenéticos en la edad adulta fueron desarrollados en ratas: un grupo madre proveía cuidado y atención hacia sus crías, y otro mostraba cuidados maternos mínimos. Se demostró que los bajos niveles de cuidado materno llevan a una disminución en el ARNm del gen que codifica para el receptor de glucocorticoides - hormona liberada cuando se activa el eje HPA- en el hipocampo de la progenie cuando es adulta; el bajo cuidado materno también se vinculó con un aumento en la metilación del ADN (Weaver, 2007; Weaver et al., 2004).

Estudios realizados en humanos han tomado un enfoque global evaluando cambios epigenéticos en miles de genes de manera simultánea. Se han analizado muestras post-mortem tomadas de la región cerebral llamada hipocampo, de sangre y de saliva tanto en adolescentes como en adultos (Essex et al., 2013; Labonté et al., 2012; Provençal et al., 2014; Tobi et al., 2009) que sufrieron una infancia adversa como crecer en un estatus socioeconómico bajo, un período de hambruna debido a una guerra, episodios de abuso sexual o niveles altos de estrés dentro del núcleo familiar. Se sugiere que hay un mayor grado de metilación del ADN en los pacientes que sufrieron una infacia de maltrato (Labonté et al., 2012), mientras que el grado de metilación es reducido en adultos cuyas madres vivieron un período de hambruna (Tobi, van den Heuvel et al., 2018); algunos trabajos indican que los patrones de metilación del ADN podrían depender del género (Essex et al., 2013; Tobi et al., 2009). Con respecto a los sitios del genoma donde ocurren los cambios epigenéticos, algunos trabajos sugieren que hay sitios en el genoma más vulnerables a almacenar esas huellas químicas (Borghol et al., 2012; Provençal et al., 2014).

Existen estudios que apoyan la propuesta de que los primeros años de vida son una etapa altamente vulnerable de sufrir cambios en la fisiología humana: se ha encontrado que los cambios epigenéticos en el adulto están mayormente vinculados con una pobre calidad de vida en la infancia que en la misma edad adulta (Borghol et al., 2012). Por otra parte, en muestras provenientes de adultos cuyas madres vivieron su embarazo durante un período de hambruna se observan cambios en la metilación de genes relacionados con el metabolismo; estas marcas de metilación se relacionan a su vez con el índice de masa corporal y con el nivel de triglicéridos en el adulto (Tobi, Slieker et al., 2018).

Modelando matemáticamente los cambios en el epigenoma —conjunto de marcas epigenéticas de to do el material genético - de embriones se ha propuesto que una reducción en la variabilidad de las huellas de metilación del ADN después de la fertilización podría ser el vínculo entre experiencias adversas en el útero y el patrón de metilación observado en el adulto (Tobi, van den Heuvel et al., 2018); así, las experiencias adversas que vive la madre durante la gestación estarían restringiendo el establecimiento de marcas de metilación del ADN en el feto. 
Recientemente se reportó que las alteraciones epigenéticas causadas por eventos adversos podrían heredarse a lo largo de generaciones. En muestras de sangre obtenidas de sobrevivientes del Holocausto judío se observó una alta metilación del gen FKBP5 (FK506 binding protein 5), el cual participa en la regulación inmune y en el plegamiento y tráfico de proteínas). De manera aún más interesante se observan cambios en la metilación de este mismo gen en los hijos de estos sobrevivientes, aunque los cambios fueron menos pronunciados (Yehuda et al., 2016), sugiriendo así una "dilución" de los cambios epigenéticos a través de generaciones, a pesar de que una huella de la información se mantiene.

\section{Conclusiones}

Una pobre calidad de vida durante el período de gestación o la infancia causa alteraciones cardiometabólicas, inmunitarias, neuroanatómicas y hormonales en el adulto. Dentro de las alteraciones psicológicas existe una mayor propensión a desarrollar depresión, ansiedad y, en general, desórdenes en el estado de ánimo. La epigénesis, que se refiere a modificaciones químicas reversibles en el ADN o histonas, lo que altera la expresión génica, se postula como uno de los mecanismos que permitirían dejar huellas en el genoma, que originarían que varios años después de un evento adverso se observen secuelas en la salud del adulto.

\section{Referencias}

- Azad, M. B., Lissitsyn, Y., Miller, G. E., Becker, A. B., HayGlass, K. T., y Kozyrskyj, A. L. (2012). Influence of socioeconomic status trajectories on innate immune responsiveness in children. PLOS ONE, 7(6). DOI: https://doi.org/10.1371/journal. pone.0038669.

* Beckett, C., Maughan, B., Rutter, M., Castle, J., Colvert, E., Groothues, C., ... SonugaBarke, E. J. S. (2006). Do the effects of early severe deprivation on cognition persist into early adolescence? Findings from the English and Romanian adoptees study. Child Development, 77(3), 696-711. Dol: https://doi.org/10.1111/j.14678624.2006.00898.x.

* Borghol, N., Suderman, M., McArdle, W., Racine, A., Hallett, M., Pembrey, M., ... Szyf, M. (2012). Associations with early-life socio-economic position in adult DNA methylation. International Journal of Epidemiology, 41(1), 62-74. Dol: https://doi. org/10.1093/ije/dyr147.

* Butchart, A., Harvey, A. P., Mian, M., y Fürniss, T. (2009). Prevención del maltrato infantil: Qué hacer, y cómo obtener evidencias. Organizacion Mundial de la Salud.

* Calkins, K. y Devaskar, S. U. (2011). Fetal Origins of Adult Disease. Current problems in pediatric and adolescent health care, 41(6), 158-176. Dol: https://doi.org/10.1016/j. cppeds.2011.01.001. 
"Epigénesis: secuelas de una infancia adversa" Sofia Gonzalez Salinas, Sergio Manuel Sánchez Moguel y Nelida Ramírez Jiménez

Cohen, R. A., Grieve, S., Hoth, K. F., Paul, R. H., Sweet, L., Tate, D., ... Williams, L. M. (2006). Early life stress and morphometry of the adult anterior cingulate cortex and caudate nuclei. Biological Psychiatry, 59(10), 975-982. Dol: https://doi. org/10.1016/j.biopsych.2005.12.016.

* Cuevas, S., Blanco, J., Juárez, C., Palma, O. y Valdez-Santiago, R. (2006). Violencia y embarazo en usuarias del sector salud en estados de alta marginación en México. Salud Publica Mex, 48 supl 2, S239-S249.

* De Bellis, M. D., Chrousos, G. P., Dorn, L. D., Burke, L., Helmers, K., Kling, M. A., ... Putnam, F. W. (1994). Hypothalamic-pituitary-adrenal axis dysregulation in sexually abused girls. The Journal of Clinical Endocrinology and Metabolism, 78(2), 249-255.

- Delgado-Coello, B. A. (2011). ¿Qué es la epigenética? Ciencia, enero-marzo, 73-82.

- Downs, W. R. y Rindels, B. (2004). Adulthood depression, anxiety, and trauma symptoms: a comparison of women with nonabusive, abusive, and absent father figures in childhood. Violence and Victims, 19(6), 659-671.

*ntringer, S., Kumsta, R., Nelson, E. L., Hellhammer, D. H., Wadhwa, P. D. y Wüst, S. (2008). Influence of prenatal psychosocial stress on cytokine production in adult women. Developmental Psychobiology, 50(6), 579-587. Dol: https://doi.org/10.1002/ dev.20316.

*ntringer, S., Wüst, S., Kumsta, R., Layes, I. M., Nelson, E. L., Hellhammer, D. H. y Wadhwa, P. D. (2008). Prenatal psychosocial stress exposure is associated with insulin resistance in young adults. American Journal of Obstetrics and Gynecology, 199(5), 498.e1-7. Dol: https://doi.org/10.1016/j.ajog.2008.03.006.

* Espinoza-Gómez, F., Zepeda-Pamplona, V., Bautista-Hernández, V., HernándezSuárez, C. M., Newton-Sánchez, O. A. y Plasencia-García, G. R. (2010). Violencia doméstica y riesgo de conducta suicida en universitarios adolescentes. Salud Publica Mex, 52, 213-219.

* Essex, M. J., Boyce, W. T., Hertzman, C., Lam, L. L., Armstrong, J. M., Neumann, S. M. A. y Kobor, M. S. (2013). Epigenetic vestiges of early developmental adversity: childhood stress exposure and DNA methylation in adolescence. Child Development, 84(1), 58-75. Dol: https://doi.org/10.1111/j.1467-8624.2011.01641.x.

* Ghosh, S., Singh, K. K., Sengupta, S. y Scaria, V. (2015). Mitoepigenetics: The different shades of grey. Mitochondrion, 25, 60-66. Dol: https://doi.org/10.1016/j. mito.2015.09.003.

* Gil Trejo, M. J., Laureano Viveros, A. y González-Salinas, S. (2018). Historia y estructura del ADN. TEPEXI Boletín Científico de la Escuela Superior Tepeji del Río, 5(10). DOI: https://doi.org/10.29057/estr.v5i10.3306.

* González-Salinas, S. [iTunes U - UAEH]. (2018, enero 12). Ácidos Nucleicos ADN y ARN. [Video]. Recuperado de: https://www.youtube.com/watch?v=NUZy4vB1taU.

* Heim, C., Newport, D. J., Heit, S., Graham, Y. P., Wilcox, M., Bonsall, R., ... Nemeroff, C. B. (2000). Pituitary-Adrenal and Autonomic Responses to Stress in Women After Sexual and Physical Abuse in Childhood. JAMA, 284(5), 592-597. DOI: https:// doi.org/10.1001/jama.284.5.592. 
"Epigénesis: secuelas de una infancia adversa" Sofia Gonzalez Salinas, Sergio Manuel Sánchez Moguel y Nelida Ramírez Jiménez

* Hernández Dávila, R. (2013). Información estadística y cualitativa sobre violencia en la niñez y en la adolescencia (delitos sexuales) en las entidades federativas. México: CEAMEG.

* Ho-Shing, O. y Dulac, C. (2019). Influences of genomic imprinting on brain function and behavior. Current Opinion in Behavioral Sciences, 25, 66-76. Dol: https://doi. org/10.1016/j.cobeha.2018.08.008.

* Iram Rizvi, S. F. y Najam, N. (2014). Parental psychological abuse toward children and mental health problems in adolescence. Pakistan Journal of Medical Sciences, $30(2), 256-260$.

- Kolb, B., Mychasiuk, R., Muhammad, A. y Gibb, R. (2013). Brain plasticity in the developing brain. Progress in Brain Research, 207,35-64. Dol: https://doi.org/10.1016/ B978-0-444-63327-9.00005-9.

* Labonté, B., Suderman, M., Maussion, G., Navaro, L., Yerko, V., Mahar, I., ... Turecki, G. (2012). Genome-wide epigenetic regulation by early-life trauma. Archives of General Psychiatry, 69(7), 722-731. Dol: https://doi.org/10.1001/ archgenpsychiatry.2011.2287.

* Lin, M.-J. y Liu, E. M. (2014). Does in utero exposure to Illness matter? The 1918 influenza epidemic in Taiwan as a natural experiment. Journal of Health Economics, 37, 152-163. Dol: https://doi.org/10.1016/j.jhealeco.2014.05.004.

* Lister, R., Mukamel, E. A., Nery, J. R., Urich, M., Puddifoot, C. A., Johnson, N. D., ... Ecker, J. R. (2013). Global epigenomic reconfiguration during mammalian brain development. Science (New York, N.Y.), 341(6146), 1237905. Dol: https://doi. org/10.1126/science.1237905.

* McCauley, J., Kern, D. E., Kolodner, K., Dill, L., Schroeder, A. F., DeChant, H. K., ... Bass, E. B. (1997). Clinical characteristics of women with a history of childhood abuse: unhealed wounds. JAMA, 277(17), 1362-1368.

* Mehta, M. A., Golembo, N. I., Nosarti, C., Colvert, E., Mota, A., Williams, S. C. R., ... Sonuga-Barke, E. J. S. (2009). Amygdala, hippocampal and corpus callosum size following severe early institutional deprivation: the English and Romanian Adoptees study pilot. Journal of Child Psychology and Psychiatry, and Allied Disciplines, 50(8), 943-951. Dol: https://doi.org/10.1111/j.1469-7610.2009.02084.x.

* Merbs, S. L., Khan, M. A., Jr, L. H., Oliver, V. F., Wan, J., Qian, J. y Zack, D. J. (2012). Cell-Specific DNA Methylation Patterns of Retina-Specific Genes. PLOS ONE, 7(3), e32602. Dol: https://doi.org/10.1371/journal.pone.0032602.

* Mochida, G. H.y Walsh, C. A. (2004). Genetic Basis of Developmental Malformations of the Cerebral Cortex. Archives of Neurology, 61(5), 637-640. Dol: https://doi. org/10.1001/archneur.61.5.637.

* Murgatroyd, C. y Spengler, D. (2011). Epigenetics of early child development. Frontiers in Psychiatry, 2, 16. Dol: https://doi.org/10.3389/fpsyt.2011.00016.

* Negele, A., Kaufhold, J., Kallenbach, L. y Leuzinger-Bohleber, M. (2015). Childhood trauma and its relation to chronic depression in adulthood. Depression Research and Treatment, 650804. DOI: https://doi.org/10.1155/2015/650804. 
"Epigénesis: secuelas de una infancia adversa" Sofia Gonzalez Salinas, Sergio Manuel Sánchez Moguel y Nelida Ramírez Jiménez

* Olaiz, G., Rojas, R., Valdez, R., Franco, A. y Palma, O. (2006). Prevalencia de diferentes tipos de violencia en usuarias del sector salud en México. Salud Publica Mex, 8 supl 2, S232-S238.

* Páramo Castillo, D. y Chávez Hernández, ana M. (2007). Maltrato y suicidio infantil en el Estado de Guanajuato. Salud Mental, 30(3), 59-67.

- Pérez-Amezcua, B., Rivera-Rivera, L., Atienzo, E. E., de Castro, F., Leyva-López, A. y Chávez-Ayala, R. (2010). Prevalencia y factores asociados a la ideación e intento suicida en adolescentes de educación media superior de la República mexicana. Salud Publica Mex, 52, 324-333.

* Provençal, N., Suderman, M. J., Guillemin, C., Vitaro, F., Côté, S. M., Hallett, M., ... Szyf, M. (2014). Association of childhood chronic physical aggression with a DNA methylation signature in adult human T cells. PLOS ONE, 9(4). DOI: https://doi. org/10.1371/journal.pone.0089839.

* Roseboom, T. J., Painter, R. C., van Abeelen, A. F. M., Veenendaal, M. V. E. y de Rooij, S. R. (2011). Hungry in the womb: what are the consequences? Lessons from the Dutch famine. Maturitas, 70(2), 141-145. Dol: https://doi.org/10.1016/j. maturitas.2011.06.017.

- Shrira, A., Shmotkin, D. y Litwin, H. (2012). Potentially Traumatic Events at Different Points in the Life Span and Mental Health: Findings From SHARE-Israel. The American journal of orthopsychiatry, 82(2), 251-259. Dol: https://doi.org/10.1111/ j.1939-0025.2012.01149.x.

- Sotomayor, O. (2013). Fetal and infant origins of diabetes and ill health: evidence from Puerto Rico's 1928 and 1932 hurricanes. Economics and Human Biology, 11(3), 281-293. DOI: https://doi.org/10.1016/j.ehb.2012.02.009.

* Springer, K. W., Sheridan, J., Kuo, D. y Carnes, M. (2007). Long-term physical and mental health consequences of childhood physical abuse: Results from a large population-based sample of men and women. Child abuse and neglect, 31(5), 517530. Dol: https://doi.org/10.1016/j.chiabu.2007.01.003.

* Taliaz, D. (2013). Skills development in infants: a possible role for widespread neurogenesis? Frontiers in Behavioral Neuroscience, 7. Dol: https://doi.org/10.3389/ fnbeh.2013.00178.

* Tamayo, T., Christian, H. y Rathmann, W. (2010). Impact of early psychosocial factors (childhood socioeconomic factors and adversities) on future risk of type 2 diabetes, metabolic disturbances and obesity: a systematic review. BMc Public Health, 10, 525. Dol: https://doi.org/10.1186/1471-2458-10-525.

Tobi, E. W., Lumey, L. H., Talens, R. P., Kremer, D., Putter, H., Stein, A. D., ... Heijmans, B. T. (2009). DNA methylation differences after exposure to prenatal famine are common and timing- and sex-specific. Human Molecular Genetics, 18(21), $4046-$ 4053. Dol: https://doi.org/10.1093/hmg/ddp353.

* Tobi, E. W., Slieker, R. C., Luijk, R., Dekkers, K. F., Stein, A. D., Xu, K. M., ... Heijmans, B. T. (2018). DNA methylation as a mediator of the association between prenatal adversity and risk factors for metabolic disease in adulthood. Science Advances, 4(1), eaao4364. Dol: https://doi.org/10.1126/sciadv.aao4364. 
* Tobi, E. W., van den Heuvel, J., Zwaan, B. J., Lumey, L. H., Heijmans, B. T. y Uller, T. (2018). Selective Survival of Embryos Can Explain DNA Methylation Signatures of Adverse Prenatal Environments. Cell Reports, 25(10), 2660-2667.e4. Dol: https:// doi.org/10.1016/j.celrep.2018.11.023.

- Tomoda, A., Suzuki, H., Rabi, K., Sheu, Y.-S., Polcari, A. y Teicher, M. H. (2009). Reduced prefrontal cortical gray matter volume in young adults exposed to harsh corporal punishment. Neurolmage, 47 Suppl 2, T66-71. Dol: https://doi. org/10.1016/j.neuroimage.2009.03.005.

Tottenham, N., Hare, T. A., Quinn, B. T., McCarry, T. W., Nurse, M., Gilhooly, T., ... Casey, B. (2010). Prolonged institutional rearing is associated with atypically larger amygdala volume and difficulties in emotion regulation. Developmental science, 13(1), 46. DOI: https://doi.org/10.1111/j.1467-7687.2009.00852.x.

* Valdez-Santiago, R. y Sanín-Aguirre, L. H. (1996). La violencia doméstica durante el embarazo y su relación con el peso al nacer. Salud Publica Mex, 38, 352-362.

* Weaver, I. C. G. (2007). Epigenetic programming by maternal behavior and pharmacological intervention. Nature versus nurture: let's call the whole thing off. Epigenetics, 2(1), 22-28.

* Weaver, I. C. G., Cervoni, N., Champagne, F. A., D'Alessio, A. C., Sharma, S., Seckl, J. R., ... Meaney, M. J. (2004). Epigenetic programming by maternal behavior. Nature Neuroscience, 7(8), 847-854. DOI: https://doi.org/10.1038/nn1276.

Yehuda, R., Daskalakis, N. P., Bierer, L. M., Bader, H. N., Klengel, T., Holsboer, F. y Binder, E. B. (2016). Holocaust exposure induced intergenerational effects on FKBP5 methylation. Biological Psychiatry, 80(5), 372-380. Dol: https://doi.org/10.1016/j. biopsych.2015.08.005.

* Zlotnick, C., Warshaw, M., Shea, M. T. y Keller, M. B. (1997). Trauma and chronic depression among patients with anxiety disorders. Journal of Consulting and Clinical Psychology, 65(2), 333-336.

\section{Cómo citar este artículo}

* Gonzalez Salinas, S., Sánchez Moguel, S. M. y Ramírezjiménez, N. (2019). Epigénesis: secuelas de una infancia adversa. Revista Digital Universitaria (RDU). Vol. 20, núm. 2 marzo-abril. Dol: http://doi.org/10.22201/codeic.16076079e.2019.v20n2.a1 